\title{
Learning Content Development in Modeling Creative Industry Objects Using Real Function Formulas Supported with Maple
}

\author{
Kusno*, Bagus Juliyanto, Kiswara Agung Santoso \\ Mathematics Department, Faculty of Mathematics and Sciences, University of Jember, Indonesia \\ ${ }^{*}$ Corresponding author. Email: kusno.fmipa@unej.ac.id
}

\begin{abstract}
Creative industries are a national strategic commodity to support international marketing. For this reason, modeling creative industry objects are essential for resulting in various shapes and features of the goods. This paper presents to develop learning content in modeling creative goods supported with the Maple software. The study method uses development research with stages as follows: (a) Instructional contents design in constructing creative objects; (b) representation techniques and evaluation of graphics for modeling the creative items; (c) Construction steps and simulation to realize the required outcomes. It founds some instructional materials and analytical formulas to motivate students to create, work and evaluate the modeling processes and results of the creative goods. The use of Maple can help them to ensure their achievements and success in presenting graphs and the simulation process. This study contributes to students recognizing and implementing mathematics formulas in modeling creative industry objects using Maple.
\end{abstract}

Keywords: Development, Learning content, Modeling, Creative object, Equation formulas, Maple software.

\section{INTRODUCTION}

Mathematical formulas were introduced to model industrial objects, mechanical components, home appliances, and creative objects shapes. It consists of designing various forms of car bodies, and electronic equipment with complex surfaces [1,2]. Implementing developable surfaces are essential for modeling the industrial goods constructed by plat-metal or playwood sheets, for example, ship hulls industries, aircraft, and trains $[3,4,5,6]$. For this reason, knowledge and skills about the formulation of curves, surfaces, and their simulations in instructional geometry modeling are essential for students to provide practical experience in designing object shapes types of creative crafts.

Objects modeling can use parametric functions or real functions in the forms of explicit or implicit equations $[7,8]$. From a curve, it can formulate many pipes form $[9,10,11,12]$. Besides, using surfaces formulation can design electronic equipment or mechanical components. This paper focuses on discussing development of learning content in creative goods modeling with real functions formulas.
The crafts and souvenirs shapes created from wood and marble have commonly unique characteristics and forms. They are generally symmetrical shape, round and solid components, closed surfaces, and many axes. In connection with this need and developing the learning materials in the analytical geometry course, we apply the real function formulas, i.e., the quadric forms, to model the creative goods. To facilitate simulation activities for students, we prefer to use the Maple tool.

This study aims to develop instructional materials for third-semester college students. The learning materials expand to create creative goods are some analytical geometry formulas. The contributions of these instructional developments are to motivate the students to learn creatively, autonomously, and responsibly in implementing mathematics formulas using Maple.

\section{METHOD}

This study applies development research, i.e., how to optimize the technology implementation in solving instructional or non-instructional learning problems [13]. In this case, we seek to identify and describe the conditions to facilitate the design and development 
processes of the instructional materials utilizing Maple $[14,15]$. The method uses the stages as follows [16]. (a) Instructional contents design in constructing creative objects; (b) Representation techniques and evaluation of graphs for modeling the creative industries; (c) Construction steps and simulation to realize the required outcomes. The use of Maple is to support the students in presenting graphs and the simulation process. This Maple tool may help and motivate the collegers to deal with how must they think, create, work and evaluate their modeling processes and results.

\section{RESULTS AND DISCUSSION}

\subsection{Instructional Contents Design in Modeling Creative Objects}

Preparing suitable instructional materials with students' knowledge, Maple software, and the designed creative objects needs to evaluate the algebraic representation of real function formulas $f(x): \mathscr{R}^{1} \rightarrow \mathscr{R}^{1}$ in $2 D$ and $g(x, y): \mathscr{R}^{2} \rightarrow \mathscr{R}^{1}$ in $3 D$. In explicit or implicit forms, the functions $\mathrm{f}$ and $\mathrm{g}$ use for drawing a curve in the plane and a surface in space, respectively, for designing the shapes of the goods and their components. Some lists of the instructional contents that are useable to construct the creative objects are as follows.

\subsubsection{Coordinate System and Axes References for Designing Objects}

The use of the coordinate system depends on the need to create the objects. This implementation is related to the size calculation of the goods, the model of its presentation, the formulation for the graph. It includes the complexity, equilibrium, or symmetry of the goods. Besides, the choice of the coordinate system often utilizes local coordinate references rather than global coordinates of the objects to facilitate modeling. For these reasons, several coordinate systems use in graphic design, namely Cartesian, polar, tubular, and spherical coordinates. In this creative object modeling, we apply the use of Cartesian coordinates. The position of a point in the plane and space presents as follows. Any point $P$ in the Cartesian plane express with notation $P(x, y)$, and in space, it represents by $P(x, y, z)$ where $x, y$, and $z$ are real numbers. On the other hand, if we have a pair of real numbers $(x, y)$ or triple of numbers $(x, y, z)$, we can find a unique point $P$ that the coordinates $P$ are $x$ and $y$ in the plane and $x, y$ and $z$ in the space (Figures $1 \mathrm{a}, 1 \mathrm{~b}$ ).

Determining axes of references implement to design the objects and their components in various branches and shapes symmetrically. We can choose the axes references in the vertical direction $\left(A_{v}\right)$, horizontal $\left(A_{H}\right)$ as shown in Figure 1c, or oblique direction relative to the Cartesian coordinates. The use of function formulas and axes references can control the height, width, volume, and shape of created goods components.
Using Cartesian coordinates system 2D and 3D in Figure 1a and Figure $1 \mathrm{~b}$ can simulate the shapes and the joints of some curves and surfaces of the real functions with Maple software. Then, it can apply to design the creative objects as showns in Figure 1c.

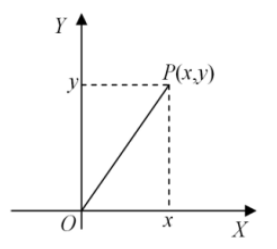

(a)

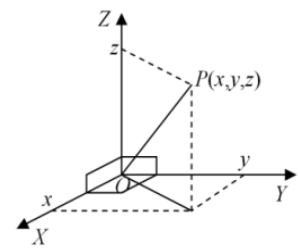

(b)

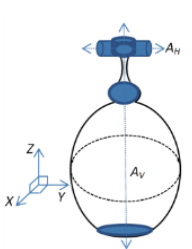

(c)
Figure 1 Coordinates and axes.

\subsubsection{Points Transformation in Space and Real Function formulas}

Consider a transformation in space $\mathrm{T}: \mathscr{R}^{3} \rightarrow \mathscr{R}^{3}$ of a point $\mathrm{P}(\mathrm{x}, \mathrm{y}, \mathrm{z})$ to the point $\mathrm{P}^{\prime}\left(\mathrm{x}^{\prime}, \mathrm{y}^{\prime}, \mathrm{z}^{\prime}\right)$ such that $\mathrm{T}(\mathrm{P})=\mathrm{P}^{\prime}$ or $\mathrm{P}^{\prime}=\mathrm{T}(\mathrm{P})$ in the matrix form $\mathrm{P}^{\prime}=\mathrm{PA}$ or

$$
\left(\begin{array}{lll}
x^{\prime} & y^{\prime} & z^{\prime}
\end{array}\right)=\left(\begin{array}{lll}
x & y & z
\end{array}\right)\left(\begin{array}{lll}
a & b & c \\
d & e & f \\
g & h & i
\end{array}\right) .
$$

Implementing the transformation of points for designing the creative objects, we can identify some transformation types based on these element values of the coefficient matrix A as follows.

1. If $a=1, b=0, c=0, d=0, e=1, f=0, g=0, h=0, i$ $=-1$, then the transformation $T$ is a reflection to the plane $X O Y$ coded $R_{X O Y}$.

2. If $a=1, b=0, c=0, d=0, e=-1, f=0, g=0, h=0, i$ $=1$, then the transformation $T$ is a reflection to the plane $X O Z$ noted $R_{X O Z}$.

3. If $a=1, b=0, c=0, d=0, e=-1, f=0, g=0, h=0, i$ $=1$, then the transformation $T$ is a reflection to the plane $X O Z$ noted $R_{X O Z}$.

4. If $a=\cos \theta, b=-\sin \theta, c=0, d=\sin \theta, e=\cos \theta, f=0$, $g=0, h=0, i=1$, then the transformation $T$ is a rotation to the axe $Z$ noted $R_{o}$.

5. If $a=k, b=0, c=, d=0, e=k, f=0, g=0, h=0$, $i=k$, then the transformation $T$ is a dilatation with scalar value $k$ coded $D_{k}$

Presenting geometry elements and shapes can use the nonparametric equation in the explicit or implicit form. Both equations types have different characteristics. The explicit equations form will generally draw an open graph for all values of the function domain, but the implicit equation can express multiple curves or closed graphs. This section discuss the real function formulas used for this discussion topic.

Consider the real functions $f(x): \mathscr{R}^{1} \rightarrow \mathscr{R}^{1}$ and $g(x, y)$ : $\mathscr{R}^{2} \rightarrow \mathscr{R}^{1}$ in a plane and space consecutively. Some 
equation formulas that define the lines, polynomial, quadric $2 D$, surfaces, and quadric $3 D$ represent in Table 1 .

Table 1. List of some equations in explicit and implicit representation

\begin{tabular}{|c|c|c|c|c|}
\hline Number & Object Name & Equation Representation & Types & Codes \\
\hline 1. & Line & $f(x)=a x+b$ with $a, b$ real values. & Explicit & $L_{E}$ \\
\hline 2. & Line & $a x+b y+c=0$ with $a, b, c$ real values. & Implicit & $L_{I}$ \\
\hline 3. & Polynomial of degree $n$ & $f(x)=a_{n} x^{n}+a_{n-1} x^{n-1}+\ldots+a_{1} x+a_{o}$ & Explicit & $P_{n}$ \\
\hline 4. & Cirle of center $(a, b)$ & $(x-a)^{2}+(y-b)^{2}=r^{2}$ with $r$ radius of circle. & Implicit & $C_{r}$ \\
\hline 5. & Ellipse of center $(a, b)$ & $\frac{(x-a)^{2}}{u^{2}}+\frac{(x-b)^{2}}{v^{2}}=1$ & Implicit & $E_{(a, b)}$ \\
\hline 6. & Hyperbole of center $(a, b)$ & $\frac{(x-a)^{2}}{u^{2}}-\frac{(x-b)^{2}}{v^{2}}=1$ & Implicit & $H_{(a, b)}$ \\
\hline 7. & Parabole & $y=a x^{2}+b x+c$ with $a \neq 0, b$, and $c$ of real numbers. & Explicit & $P_{a}$ \\
\hline 8. & Sphere of center $(a, b, c)$ & $(x-a)^{2}+(y-b)^{2}+(z-c)^{2}=r^{2}$. & Implicit & $S_{p}$ \\
\hline 9. & Ellipsoid of center $(a, b, c)$ & $\frac{(x-a)^{2}}{u^{2}}+\frac{(y-b)^{2}}{v^{2}}+\frac{(z-c)^{2}}{w^{2}}=1$ & Implicit & $E_{d}$ \\
\hline 10. & Hyperboloid & $\frac{x^{2}}{u^{2}}+\frac{y^{2}}{v^{2}}-\frac{z^{2}}{w^{2}}=1$ & Implicit & $H_{d}$ \\
\hline 11. & Cone & $a x^{2}+b y^{2}=(c-z)^{2}$ with $a, b$, and $c$ real numbers. & Implicit & $C_{n}$ \\
\hline 12. & Paraboloid & $a x^{2}+b y^{2}+c=z$ with $a, b$, and $c$ real numbers. & Implicit & $P_{d}$ \\
\hline 13. & Surface & $g(x, y)=\mathrm{z}$ & Explicit & $S_{u}$ \\
\hline
\end{tabular}

\subsection{Representation Techniques and Evaluation of Graphs Using Maple}

This section discusses the various uses and evaluates some advantages of the equations listed in the third column of Table 1 that are ordered from the number 1 up to 13 in the first column of that table. These equations will be plotted in Maple software [17,18] to construct the creative objects. Then, we explain how to create more complex graphs by joining some types of curve or surface segments.

The linear function $f(x)=a x+b$ with $a, b$ real values of formula (1) in Table 1 can draw a line segment defined by two points $P\left(x_{P}, y_{P}\right)$ and $Q\left(x_{Q}, y_{Q}\right)$ of interval domain $x_{P} \leq x \leq x_{Q}$ with horizontal or oblique directions. In contrast, the line equation $a x+b y+c=0$ with $a, b, c$ real values use to design a line segment of endpoints $P\left(x_{P}, y_{P}\right)$ and $Q\left(x_{Q}, y_{Q}\right)$ in horizontal, vertical, or oblique directions and the interval domain $x_{P} \leq x \leq x_{Q}$ and $y_{P} \leq y \leq y_{Q}$. The example formulas of these line segments are respectively shown in Figure 2a and 2b using Maple formula (1) and (2) in Table 2.

On the other sides, we can generate the polynomial of degree $n$ by employing $n+1$ different points. In this case, formulating cubic and quartic polynomial in Figure 2c and Figure 2d with Maple tool is shown in Table 2.

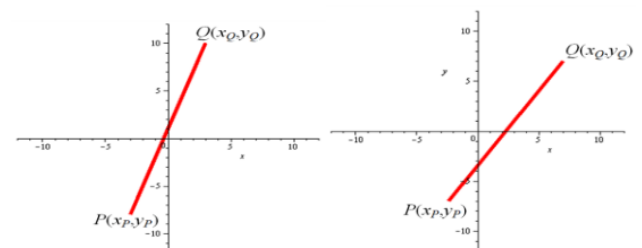

(a)

(b)

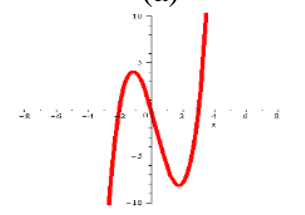

(c)

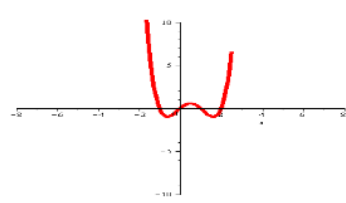

(d)
Figure 2 Lines and polynomials presentation.

The shapes modification and the shifting graph positions of the quadric formulas 4 up to the formula (11) in Table 1 principally depend on determining parameter values and domain interval of these equations. It consists of the choice of the coefficients, the quadric centers parameter, and the radius parameters of the equations. Moreover, it can also model the curve and surface shapes of the quadric objects by limiting different domain intervals of variables $x, y$, or $z$. Figure 3 a presents an ellipse $\frac{(x-a)^{2}}{u^{2}}-\frac{(x-b)^{2}}{v^{2}}=1$ with $a=3, b=2, u=3, v=$ 4 , and $-3 \leq x, y \leq 8$. Figure $3 b$ illustrates a hyperboloid $\frac{x^{2}}{u^{2}}+\frac{y^{2}}{v^{2}}-\frac{z^{2}}{w^{2}}=1$ with $u=1, v=\sqrt{ } 3, w=2$, and $-6 \leq$ $x \leq 6,-6 \leq y \leq 3$, and $-6 \leq z \leq 6$. Then, Figure $3 c$ presents a paraboloid: $z=a x^{2}+b y^{2}+c$ with $a=1, b=2, c=-4$, - 
$2 \leq x \leq 3,-6 \leq y \leq 6$, and $-6 \leq y \leq 6$. Formula syntaxes of Maple for these equations can find in Table 2 from Figure $3 \mathrm{a}$ up to $3 \mathrm{c}$.

The surface $g(x, y)=z$ can use to define the planes with different tangents and create a surface patch with various arches in direction $x, y$ or free forms. If we elect the functions $g_{1}(x, y)=z=3 x+4 y, g_{2}(x, y)=z=3 y$, and $g_{3}(x, y)=z=-x+2 y+2$, with $-10 \leq x, y \leq 10$, then it finds the graph as shown in Figure 3d. The graph $g_{4}(x, y)=z=$ $x^{3}-x^{2}-6 x$ with $-12 \leq x, y \leq 12$ and $-20 \leq x, y \leq 20$ has two arches in direction y Figure $3 \mathrm{e}$, meanwhile the formula $g_{5}(x, y)=z=x y^{2}+x^{3} y$, with $-12 \leq x, y \leq 12$ is free form surface Figure $3 \mathrm{f}$. Formula syntaxes of Maple for these graphs can find in Table 2 from Figure $3 d$ up to $3 f$.

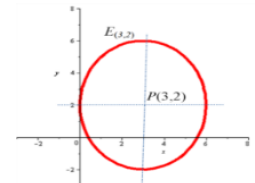

(a)

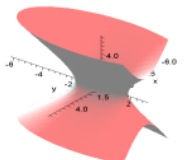

(b)

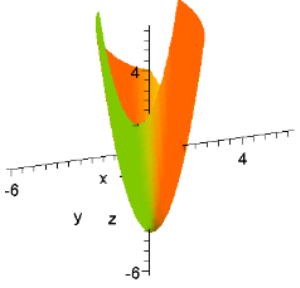

(c)

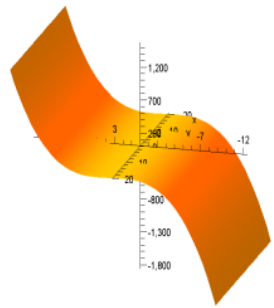

(e)

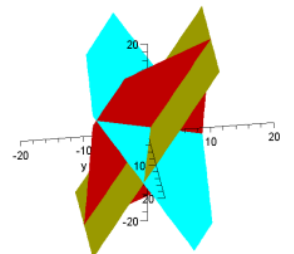

(d)

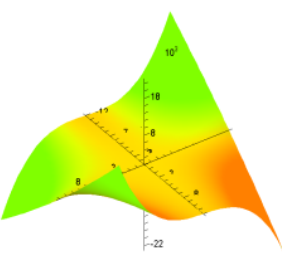

(f)
Figure 3 Quadric form of a curve and the surfaces presentation.

Table 2. Plotting the graphs using Maple

\begin{tabular}{|c|c|c|c|}
\hline Number & \multicolumn{2}{|c|}{ Equations Representation of Figure 2 and 3 Using Maple } & Explanations \\
\hline 1. & \multicolumn{2}{|c|}{$\begin{array}{l}>\operatorname{with}(\text { plots }): \\
>\operatorname{plot}\left(3^{*} x+1, x=-3 . .3, \text { thickness }=5, \text { view }=[-12 . .12,-12 . .12]\right)\end{array}$} & Figure $2 \mathrm{a}$ \\
\hline 2. & \multicolumn{2}{|c|}{$\begin{array}{l}>\text { implicitplot }(-3 * x+2 . * y=-7, x=-8 . .8, y=-7 . .7 \text {, thickness }=5 \text {, } \\
\quad \text { view }=[-12 . .12,-12 . .12])\end{array}$} & Figure $2 b$ \\
\hline 3. & \multicolumn{2}{|c|}{$>\operatorname{plot}\left(x^{\wedge} 3-x^{\wedge} 2-6 * x, x=-5 . .5\right.$, thickness $=5$, view $\left.=[-8 \ldots 8,-10 \ldots 10]\right)$} & Figure $2 c$ \\
\hline 4. & \multicolumn{2}{|c|}{$\begin{array}{l}>\operatorname{plot}\left(x^{\wedge} 4-2 * x^{\wedge} 3-x^{\wedge} 2+2 * x, x=-2 . .2 .5, \text { thickness }=5\right. \\
\text { view }=[-8 . .8,-10 \ldots 10])\end{array}$} & Figure $2 d$ \\
\hline 5. & \multicolumn{2}{|c|}{$\begin{array}{l}>\text { implicitplot }\left((1 / 9)^{*}(x-3)^{\wedge} 2+(1 / 16)^{*}(y-2)^{\wedge} 2=1, x=-3 . .8, y=-3 . .8,\right. \\
\text { thickness }=5, \text { view }=[-3 . .8,-3 . .8])\end{array}$} & Figure $3 a$ \\
\hline 6. & \multicolumn{2}{|c|}{$\begin{array}{l}>\text { implicitplot } 3 \mathrm{~d}\left(x^{\wedge} 2+(1 / 3)^{*} y^{\wedge} 2-(1 / 4)^{*} z^{\wedge} 2=1, x=-6 . .6, y=-6 . .3\right. \\
\quad \mathrm{z}=-6 . .6, \text { grid }=[50,50,50]) ;\end{array}$} & Figure $3 b$ \\
\hline 7. & \multicolumn{2}{|c|}{$>$ implicitplot $3 \mathrm{~d}\left(x^{\wedge} 2+2^{*} y^{\wedge} 2-4=z, x=-3 . .2, y=-6 . .6, z=-6 . .6\right.$} & Figure $3 c$ \\
\hline 8. & \multicolumn{2}{|c|}{$\begin{array}{l}g 2:=\operatorname{plot} 3 \mathrm{~d}\left(2^{*} y, x=-10 . .10, \mathrm{y}=-10 . .10, \text { color }=\text { yellow }\right) \\
g 3:=\operatorname{plot} 3 \mathrm{~d}\left(-x-2^{*} y+2, x=-10 . .10, \mathrm{y}=-10 \ldots 10, \text { color }=\text { cyan }\right) \\
\text { display }(g 1, g 2, g 3, \text { view }=[-20 . .20,-20 . .20,-20 . .20], \text { grid }=[50,50])\end{array}$} & Figure $3 d$ \\
\hline 9. & \multicolumn{2}{|c|}{$\begin{array}{l}>g 4:=\operatorname{plot} 3 \mathrm{~d}\left(x^{\wedge} 3-x^{\wedge} 2-6^{*} x, x=-12 . .12, y=-20 . .20, \text { grid }=[49,49]\right) \\
\quad \text { display }(g 4)\end{array}$} & Figure $3 \mathrm{e}$ \\
\hline 10. & \multicolumn{2}{|c|}{$\begin{array}{l}>g 5:=\operatorname{plot} 3 \mathrm{~d}\left(x^{*} y^{\wedge} 2+x^{\wedge} 3^{*} y, x=-12 . .12, y=-12 . .12, \text { grid }=[49,49]\right) \\
\text { display }(g 5)\end{array}$} & Figure $3 \mathrm{f}$ \\
\hline \multicolumn{2}{|c|}{$\begin{array}{l}\text { Connecting some curves or surfaces pieces can create } \\
\text { re complex curves or patches (small parts of the } \\
\text { face) that offer for designing the features of the } \\
\text { nponents of the crafts goods and souvenirs. Joining } \\
\text { curve segments need to calculate a common } \\
\text { andary point and tangent line of both curves. } \\
\text { nnecting two adjacent patches need to compute a } \\
\text { nmon boundary curve and the tangent plane of both } \\
\text { ches. We call these conditions continuity of zero } \\
\text { grees }\left(C^{0}\right) \text { and one degree }\left(C^{1}\right) \text {. Two curves } f_{11}(x)=2 x \\
\text { with } x \text { in interval }-10 \leq x \leq 2 \text { and } f_{12}(x)=-x 2+x+\end{array}$} & \multicolumn{2}{|c|}{$\begin{array}{l}10 \text { with } x \text { in interval } 2 \leq x \leq 10 \text { are continuous } C^{0} \text { at the } \\
\text { point } P(2,8) \text {, because } f_{11}(2)=f_{12}(2)=8 \text { as presented in } \\
\text { Figure } 4 \mathrm{a} \text {. The curves } f_{21}(x)=3 x^{3}+x^{2}+x-1 \text { with } x \text { in } \\
\text { interval }-6 \leq x \leq 1 \text { and } f_{22}(x)=x^{3}+x^{2}+7 x-5 \text { with } x \text { in } \\
\text { interval } 1 \leq x \leq 6 \text { are continuous } C^{1} \text { at the point } P(1,4) \text {, } \\
\text { because } f_{21}(1)=f_{22}(1)=4 \text { and } f_{21}^{\prime}(1)=f_{22}^{\prime}(1)=12 \text { in } \\
\text { Figure } 4 \mathrm{~b} \text {. On the other hand, the surface equations } \\
f_{31}(x, y, z): x^{2}+y^{2}=66 \text { and } f_{32}(x, y, z): x^{2}+y^{2}+(z-20)^{2}=66 \\
\text { with }-9 \leq x, y \leq 9 \text { as illustrated in Figure } 4 \mathrm{c} \text { are continuous } \\
\text { along the common boundary curve } x^{2}+y^{2}=66 \text { at } z=20 \text {, } \\
\text { because } f_{31}(x, y, 20)=f_{31}(x, y, 20)=x^{2}+y^{2}-66 \text {. The tangent }\end{array}$} \\
\hline
\end{tabular}


planes at any points along the common boundary curve $x^{2}+y^{2}=66$ at $z=20$ of both surfaces are unique. The joint between these surface pieces at $z=20$ is presented in Figure 4d.

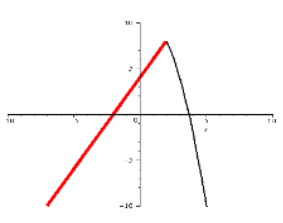

(a)

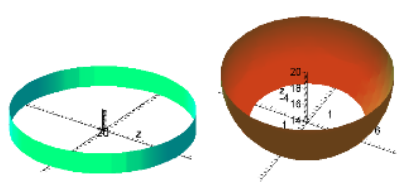

(c)

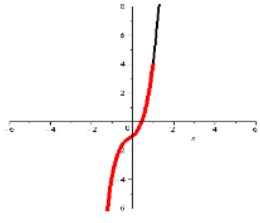

(b)

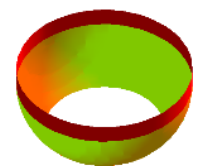

(d)
Figure 4 Connection between curves and surfaces.

It can summarize that some equation formulas of Table 1 may offer various curves and surfaces shapes that can model the component forms of the creative objects. Then, joining the parts of the objects will construct these required goods. Based on these results, in the next section, we study the construction of the creative goods by implementing the equations in Table 1 and the point transformation formulas (1).

\subsection{Construction Steps and Simulation}

Designing shapes and reliefs of the creative objects that include handicraft [19], gifts, and cottage industry often hold with trial and error ways manually. On the other hand, using formulas 1 up to 12 listed in Table 1 and objects axes laid in Cartesian coordinate with the Maple tool can plot the shapes and features of the goods with these steps.

a. Determine the object axes to control the height, volume, and position of goods components.

b. Formulate visual forms of each required part of the goods using among formulas in Table 1. c. Compute points or curves that can continuously share the joints between two object components adjacent.

d. Choose a style, color, or lighting using the Maple software menu to show this object naturally.

In designing Figure 5b, first, it determines a vertical axes $A_{v}$ as shown in Figure 5a. Then, from the bottom of the axes, we orderly prepare the heights $t_{1}, t_{2}, \ldots, t_{6}$ to construct each component of the creative object. Based on the axes reference $A_{v}$, in the height $t_{l}$, it draws a piece of the plane using the Maple formula $P 1$ in Table 3 . After that, from the heights $t_{2}$ up to $t_{6}$, we consecutively calculate the continuous connection $C^{0}$ or $C^{l}$ between two patches adjacent $P 2$ up to $P 6$ that create the surfaces of the components of the object. Then, it plots each part of the item using the Maple formula P2 up to P6 in Table 3. Giving different colors and lighting on these components pictures with Maple menu obtain Figure $5 b$.

Figure 5c uses the vertical axes $A_{v}$ and two horizontal axes $A_{H-1}$ and $A_{H-2}$. We can reflect the object components laid in horizontal axes $A_{H-2}$ to the Plane $X O Z$, i.e., from the position $R_{X O Z-I}$ to the position $R_{X O Z-2}$ and the horizontal axes $A_{H-1}$ to the Plane $Y O Z$ to obtain symmetry forms. Using these steps of construction gets Figure $5 \mathrm{~d}$.

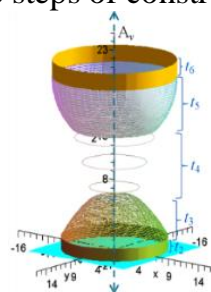

(a)

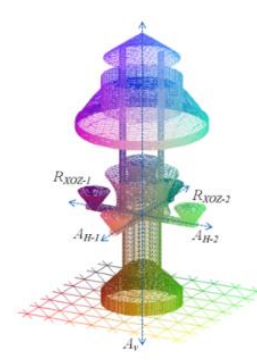

(c)

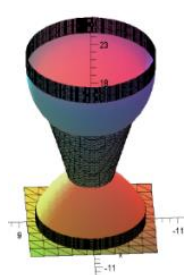

(b)

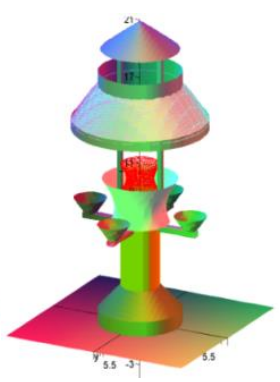

(d)
Figure 5 Creative industry goods modelling.

Table 3. Formulations of the creative objects using Maple

\begin{tabular}{|c|c|}
\hline Number & Equations Representation of Figure 5b Using Maple \\
\hline 1. & $\begin{array}{l}>\text { with(plots): } \\
>P 1:=\text { implicitplot } 3 \mathrm{~d}(z=0, x=-8 . .8, y=-8 . .8, z=0 . .2) \text { : }\end{array}$ \\
\hline 2. & $>P 2:=$ implicitplot $3 \mathrm{~d}\left(x^{\wedge} 2+y^{\wedge} 2=48, x=-8 . .8, y=-8 . .8, z=0 . .1 .5\right.$, grid $\left.=[20,20,20]\right):$ \\
\hline 3. & $>P 3:=$ implicitplot $3 \mathrm{~d}\left(\left(\mathrm{x}^{\wedge} 2+\mathrm{y}^{\wedge} 2\right) /(9.1)=6.8-\mathrm{z}, x=-8 . .8, y=-8 . .8, z=1.5 . .5 .9\right.$, grid $\left.=[20,20,20]\right):$ \\
\hline 4. & $\begin{aligned}>P 4:= & \text { implicitplot } 3 \mathrm{~d}\left(x^{\wedge} 2 /(3.45)+y^{\wedge} 2 /(3.45)-(1 / 25)^{*} z^{\wedge} 2=1, x=-6 . .6, y=-6 . .6, z=5.9 . .14,\right. \\
& \text { grid }=[20,20,20]):\end{aligned}$ \\
\hline 5. & $\begin{aligned}> & P 5:=\operatorname{implicitplot} 3 \mathrm{~d}\left(x^{\wedge} 2+y^{\wedge} 2+(z-20)^{\wedge} 2=66, x=-9 . .9, y=-9 . .9, z=14 . .20, \text { grid }=[20,20,20]\right): \\
> & P 6:=\operatorname{implicitplot} 3 \mathrm{~d}\left(x^{\wedge} 2+y^{\wedge} 2=66, x=-9 . .9, y=-9 . .9, z=20 . .22, \text { grid }=[20,20,20]\right): \\
& \operatorname{display}(P 1, P 2, P 3, P 4, P 5, P 6, \text { view }=[-11 . .9,-11 . .9,-2 . .25])\end{aligned}$ \\
\hline
\end{tabular}




\section{CONCLUSIONS}

These study results provide contributions develop the learning contents to implement and practice some real functions formulas for designing forms and features of the components of the creative object supported with Maple software. Using Maple for modeling these goods can motivate the students to participate, recognize and implement the mathematics formulas. The students will try to visualize and design these industrial objects in natural ways. The introduced steps of these goods constructions help the collegers to learn creatively, visually, autonomously, and responsibly in implementing mathematics formulas and designing the required creative industry objects

\section{REFERENCES}

[1] M.E. Mortenson, Geometry Modeling, Jonh Wiley and Sons Inc., New York, 1996.

[2] F. Yamaguchi, Curves and Surfaces in Computer Aided Geometric Design, Springer-Verlag, Berlin, 1988.

[3] L. Fernández-Jambrina, F. Pérez-Arribas, Developable Surface Patches Bounded by NURBS Curves, J. Comp. Math., vol 38(-), 2020, pp. 715-731. DOI:10.4208/jcm.1904-m20180209

[4] Kusno, Modeling of developable surfaces using Hermite spline interpolation curves, Advances in Mathematics: Scientific Journal, vol. 9(10), 2020, pp. 8431-8442. DOI: https://doi.org/10.37418/ amsj.9.10.72

[5] Kusno, Fitting a curve, cutting surface, and adjusting the shapes of developable Hermite patches, Mathematics and Statistics, vol. 8 (6), 2020, pp. 740-746. DOI: https://doi.org/10.13189/ms.2020.080615

[6] Kusno, On the modeling of developable hermite patches, Journal of Mathematical and Computational Science, vol. 11(2), 2021, pp.1145-1165. DOI: https://doi.org/10.28919/ jmcs $/ 5293$

[7] D. Salomon, Curves and Surfaces for Computer Graphics, Springer Science+Business Media, Inc., 233 Spring St., New York, 2006.

[8] A.J.P. Gomes, I.V.J. Jorge, B. Wyvill, C. Galbraith, Implicit Curves and Surfaces: Mathematics, Data Structures and Algorithms, Springer-Verlag, London Limited, 2009.
[9] K. Malaček, Z. Šibrava, Blending Circular Pipes With a Cyclic Surface, Journal for Geometry and Graphics, vol. 10(1), 2006, pp. 99-107.

[10] Kusno, A. C. Prihandoko, Continuous connection of two adjacent pipe parts defined by line, Bézier and Hermit center curves, Journal of Physics Conference Series, vol. 1008(1), 2018, pp. 1-7. DOI: https://doi.org/10.1088/1742-6596/1008/1/ 012005

[11] Kusno, Modeling pipes using pipes' center curves of quadratic and cubic spline interpolation, AIP Conference Proceedings, vol. 2329, 2021. DOI: https://doi.org/10.1063/5.0042248

[12] Kusno, On the modeling of cross-section and longitudinal section of pipes, Journal of Physics Conference Series, vol. 321(2), 2019, pp. 1-8. DOI: https://doi.org/10.1088/1742-6596/1321/2/ 022068

[13] Igi-global, What is Design and Development Research; Retrieve 17th July 2021 from https://www.igi-global.com/dictionary/designand-development-research/51339.

[14] R. C. Richey, J. D. Klein, Developmental Research Methods: Creating Knowledge from Instructional Design and Development Practice, Journal of Computing in Higher Education Spring, vol. 16(2), 2005, pp. 23-38.

[15] R. C. Richey, J. D. Klein, Design and Development Research, Handbook of Research on Educational Communications and Technology, vol. (141-150), Springer Science+Business Media New York, 2014. DOI: 10.1007/978-1-4614 3185-5_12

[16] Institute of Education Sciences (IES), Common Guidelines for Education Research and Development, A Report from the Institute of Education Sciences, U.S. Department of Education and the National Science Foundation, 2013, Retrieve 17th July 2021 from https://ies.ed.gov/pdf/ commonguidelines.pdf

[17] Maplesoft, User Manual, Waterloo Maple Inc, Canada, 2021.

[18] M. L. Abell, J. P. Braselton, Maple by Example, Elsevier Inc, Amsterdam, 2005.

[19] Kusno, I. H. Agustin, I. Halikin, On the modeling of the object surface reliefs of marble handicrafts using quartic curves and circles, IOP Conf. Series: Earth and Environmental Science, vol.243, 2019. DOI: https://doi.org/10.1088/ 1755 1315/243/1/012017 